\title{
Subdirectly irreducible Rees matrix semigroups
}

\section{David E. Zitarelli}

\begin{abstract}
Minimal congruences on a Rees matrix semigroup $S$ having at least one proper congruence are described. Necessary and sufficient conditions for $S$ to be subdirectly irreducible are given in two cases according to whether the structure group of $S$ is trivial.
\end{abstract}

\section{Introduction}

Congruences on a Rees matrix semigroup (or a completely 0-simple semigroup) have been described in various ways. The aim of this paper is to show that the recent characterization by Lallement [2] in terms of admissible triples can be used to solve a problem which the other descriptions did not seem to permit. Namely, we will give necessary and sufficient conditions for a Rees matrix semigroup to be subdirectly irreducible; that is, to have the least nontrivial congruence.

Section 2 contains several properties of admissible triples and a restatement of Lallement's Theorem. Our results on subdirect irreducibility are contained in Section 3. Obviously every congruence-free semigroup is subdirectly irreducible, so congruence-free Rees matrix semigroups are described first. Next we list the three possible forms of minimal congruences on a Rees matrix semigroup $S$ which is not congruencefree. Then we determine when $S$ is subdirectly irreducible in terms of the sandwich matrix, when the structure group $G$ is trivial, and in terms of reductivity and the subdirect irreducibility of $G$ when $G$ is nontrivial.

Received 13 December 1976. 
All undefined terms and notation can be found in [4].

\section{Admissibility}

Let $S=M^{\circ}(I, G, M ; P)$ be a regular Rees matrix semigroup. We will define an admissible triple on $S$ and relate this concept to the reductivity of $S$ and the entries of $P$.

DEFINITION. Let $r$ be an equivalence relation on $I, N$ be a normal subgroup of $G$, and $\pi$ be an equivalence relation on $M$. Then $(r, N, \pi)$ is called an admissible triple on $S$ if the following conditions are satisfied:

(A1) if irj then for all $\mu \in M, p_{\mu i} \neq 0$ implies $p_{\mu j} \neq 0$;

(A2) if irj, $p_{\mu i} \neq 0$, and $p_{\nu i} \neq 0$, then

$$
p_{\mu i} p_{\nu i}^{-1} p_{\nu j} p_{\mu j}^{-1} \in N
$$

(A3) if $\mu \pi v$ then for all $i \in I, p_{\mu i} \neq 0$ implies $p_{v i} \neq 0$;

(A4) if $\mu \pi \nu, p_{\mu i} \neq 0$, and $p_{\nu j} \neq 0$, then

$$
p_{\mu i} p_{v i}^{-1} p_{v j} p_{\mu j}^{-1} \in N
$$

For any set $A$ we will denote the identity relation by $\varepsilon_{A}$ and the universal relation by $\omega_{A}$. Where no ambiguity exists we will omit the subscripts. Also if $a, b \in A, a \neq b$, we will let $R(a, b)$ denote the equivalence relation whose only nontrivial class is the set $\{a, b\}$.

Our first two results will be fundamental to later considerations. Their proofs follow imediately from the admissibility conditions.

LEMMA 1. Let $(r, N, \pi)$ be an admissible triple on $S$. If $r^{\prime} \subseteq r, N \subseteq N^{\prime}$, and $\pi^{\prime} \subseteq \pi$ then $\left(r^{\prime}, N^{\prime}, \pi^{\prime}\right)$ is also admissible.

LEMMA 2. For each normal subgroup $N$ of $G$ the triple $(\varepsilon, N, \varepsilon)$ is admissible.

Recall that the $i$ th and $j$ th columns of the sandwich matrix $P$ are right proportional if there exists some element $e \in G$ such that $p_{\mu i}=p_{\mu j}^{c}$ for all $\mu \in M$. 
LEMMA 3. If $(r, e, \varepsilon)$ is admissible for some $r \neq \varepsilon_{I}$ then two distinct colums of $P$ are right proportional.

Proof. Since $r \neq \varepsilon_{I}$ there exist $i \neq j \in I$ such that $i r j$. We will show that the $i$ th and $j$ th columns of $P$ are right proportional.

Since $P$ is regular, $p_{v i} \neq 0$ for some $\nu \in M$. Then $p_{v j} \neq 0$ by (AI) so put $c=p_{v i}^{-1} p_{v j}$. Let $\mu \in M$. If $p_{\mu i} \neq 0$ then (A2) implies that $p_{\mu i} p_{\nu i}^{-1} p_{\nu j} p_{\mu j}^{-1}=e$ whence $p_{\mu i}^{-1} p_{v j}=p_{v i}^{-1} p_{v j}=c$. On the other hand if $p_{\mu i}=0$ then $p_{v i}=0$ by (Al). Hence $p_{\mu j}=p_{\mu i} c$ for all $\mu \in M$; so the $i$ th and $j$ th columns have the desired property.

LEMMA 4. If the $i$ th and $j$ th colums of $P$ are right proportional then $(R(i, j), N, \varepsilon)$ is an admissible triple for each normal subgroup $N$ of $G$.

Proof. By hypothesis there exists some $c \in G$ such that $p_{\mu i}=p_{\mu j} c$ for all $\mu \in M$, so (Al) obviously holds. If $p_{\mu i} \neq 0$ and $p_{\nu i} \neq 0$ then $p_{\mu i} p_{v i}^{-1} p_{\nu j} p_{\mu j}^{-1}=\left(p_{\mu j} c\right)\left(p_{v j}\right)^{-1} p_{\nu j} p_{\mu j}^{-1}=e$, hence (A2) holds. The remaining admissibility conditions are easy to verify.

It is well-known (for example, [4, Theorem v.3.14]) that $S$ is left reductive if and only if no two distinct columns of $P$ are right proportional. Hence

COROLLARY 5. The following conditions are equivalent on $S$;

(i) $S$ is not left recuctive;

(ii) $(R(i, j), e, \varepsilon)$ is admissible for some $i \neq j$;

(iii) the ith and $j$ th colums of $P$ are right proportional for some $i \neq j$.

Denote the lattice of congruences on a semigroup $S$ by $C(S)$. A congruence $\sigma \in C(S)$ is called proper if it is different from the universal relation. Put

$$
C^{\prime}(S)=\{\sigma \in C(S): \sigma \neq \varepsilon \text { and } \sigma \neq \omega\} .
$$

We will conclude this section by stating the very basic result of 
Lallement [2] linking congruences on $S$ to admissible triples. The notation introduced will be used throughout the remainder of this paper.

THEOREM 6 (Lallement). Let $S=M^{0}(I, G, M ; P)$. If $(r, N, \pi)$ is an admissible triple on $S$ then the relation $\theta=\theta(r, N, \pi)$ defined on $S$ by

$$
\begin{aligned}
& (i, a, \mu) \theta(j, b, \nu) \text { iff } a \neq 0, b \neq 0, \quad i r j, \mu \pi \nu \text {, and } \\
& p_{\alpha i} a p_{\mu k} \equiv p_{\alpha j} b p_{\nu k}(\bmod N) \text { for some } \alpha \in M, k \in I \text { such that } \\
& p_{\alpha i} \neq 0, p_{\mu k} \neq 0,0 \theta 0,
\end{aligned}
$$

is a proper congruence on $S$. Conversely every proper congruence on $S$ can be written in the form $\theta(r, N, \pi)$ for some admissible triple $(r, N, \pi)$.

It can easily be verified that $\theta(r, N, \pi) \subseteq \theta(s, K, \rho)$ if and only if $r \subseteq s, N \subseteq K$, and $\pi \subseteq \rho$. Moreover, using Lemma 2 we see that $\theta_{N}=\theta(\varepsilon, N, \varepsilon) \in C(S)$ for every normal subgroup $N$ of $G$.

\section{Subdirect irreducibility}

In this section we make use of Lallement's Theorem to find all subdirectly irreducible Rees matrix semigroups. Recall that a semigroup is congruence-free if $C^{\prime}(S)=\emptyset$. (The term h-simple was used in [5].) We first dispose of those Rees matrix semigroups which are congruence-free since they are always subdirectly irreducible. For those which are not congruence-free we will consider two cases according to whether the structure group is trivial. First we will use the above results to give an alternative proof of a result due to Munn ([3, Theorem 2.1]; see also [6]).

THEOREM 7. A Rees matrix semigroup $S=M^{0}(I, G, M ; P)$ is congruence-free if and only if

(1) $G$ is a simple group and $S \simeq G$ or

(2) $G$ is the trivial group and no two distinct rows or colums of $P$ are identical.

Proof. Let $S$ be congruence-free. It follows from Lemma 2 that $\theta=\theta(\varepsilon, G, \varepsilon) \in C(S)$, so $\theta=\omega$ or $\theta=\varepsilon$. The former case implies 
that $|I|=|M|=1$. Since $\theta(\varepsilon, N, \varepsilon) \in C(S)$ for every normal subgroup $N$ of $G$ it follows that $G$ is simple. From the latter case we see immediately that $G$ is the trivial group, and that $P$ is of the desired form follows from Lemma 3 .

That such semigroups are congruence-free is obvious.

We will now proceed to describe those subdirectly irreducible Rees matrix semigroups $S$ which are not congruence-free. First we will characterize their minimal congruences.

LEMMA 8. A proper congmence $\sigma$ on $S$ is minimal if and only if $\sigma$ has one of the following three forms:

(1) $\sigma=\theta(R(i, j), e, \varepsilon)$ for some $i, j \in I, i \neq j ;$

(2) $\sigma=\theta(\varepsilon, e, R(\mu, \nu))$ for some $\mu, \nu \in M, \mu \neq \nu$;

(3) $\sigma=\theta_{N}$ for some minimal normal subgroup $N$ of $G$.

Proof. Let $\sigma=\theta(r, N, \pi)$ be minimal on $S$. Since $\theta_{N} \in C(S)$ and $\theta_{N} \subseteq \sigma$ we have either $\sigma=\theta_{N}$ or $N=e$. Thus all minimal congruences on $S$ are of the form $\theta(\varepsilon, N, \varepsilon)$ or $\theta(r, e, \pi)$.

First we show that $\theta_{N}$ is minimel if and only if $N$ is minimal. Suppose $\theta_{N}$ be minimal. If $K$ is a normal subgroup of $G$ and $K \subseteq N$ then $\theta_{K} \subseteq \theta_{N}$ so minimality implies $\theta_{K}=\varepsilon_{S}$ or $\theta_{K}=\theta_{N}$. Thus $K=e$ or $K=N$, respectively, so $N$ is minimal. Conversely if $N$ is minimal and $\sigma=\theta(r, K, \pi) \subseteq \theta_{N}$ then $r=\pi=\varepsilon$ and $K \subseteq N$. The last inclusion implies that $K=e$ or $K=N$, so $\sigma=\varepsilon_{S}$ or $\sigma=\theta_{N}$ respectively. Hence $\theta_{N}$ is minimal.

It remains to determine when $\sigma=\theta(r, e, \pi)$ is minimal. Let $\sigma$ be minimal and suppose that $r \neq \varepsilon$. Then $\theta(r, e, \varepsilon) \in C^{\prime}(S)$ by Lemma 1 , and $\theta(r, e, \varepsilon) \subseteq \sigma$, so $\pi=\varepsilon$. Further, $i r j$ for some $i \neq j \in I$, so $\theta(R(i, j), e, \varepsilon) \subseteq \theta(r, e, \varepsilon)=\sigma$. But then the minimality of $\sigma$ implies $R(i, j)=r$. Thus $\sigma=\theta(r(i, j), e, \varepsilon)$; similarly $\pi \neq \varepsilon$ implies $\sigma=\theta(\varepsilon, e, R(\mu, \nu))$ for some $\mu \neq \nu \in M$. Since $\sigma \neq \varepsilon$ we must have either $r \neq \varepsilon$ or $\pi \neq \varepsilon$, so $\sigma$ is of the desired form. That such congruences are minimal is obvious. 
The standard way of introducing subdirectly irreducible semigroups is via the direct product (see, for example, [5]). However it suits our purposes here to adopt the definition that a semigroup is subdirectly irreducible if the intersection of any set of nonidentical congruences is nonidentical.

THEOREM 9. The following conditions are equivalent on a Rees matrix semigroup $T=M^{0}(I, e, M ; P)$ over the trivial group:

(i) $T$ is subdirectly irreducible;

(ii) exactly two distinct rows or two distinct colums of $P$ are identical;

(iii) $T$ has precisely one congruence different from $\varepsilon$ and $\omega$.

Proof. (i) implies ( $i i)$. If $T$ is subdirectly irreducible with least congruence $\sigma$, then according to Lemma 8 we can say without loss of generality that $\sigma=\theta(R(i, j), e, \varepsilon)$ for some $i \neq j$. It follows from Corollary 5 that the $i$ th and $j$ th columns of $P$ are identical. Suppose two other columns of $P$, say the $k$ th and $\tau_{\text {th }}$ columns, are also identical. Using Corollary 5 again, put $\rho=\theta(R(k, l), e, \varepsilon) \in C(T)$. If $\{i, j\} \neq\{k, z\}$ then $\sigma \cap \rho=\varepsilon_{T}$. But $T$ is subdirectly irreducible and $\sigma \neq \varepsilon_{T}$, so $\rho=\varepsilon_{T}$. This means that $k=2$. A similar approach shows that no other column of $P$ is equal to either the $i$ th or $j$ th column, hence these are the only distinct identical columns of $P$.

Now suppose that two rows, say the $\mu$ th and $\nu$ th rows, are equal. Then Corollary 5 implies that $\tau=\theta(\varepsilon, e, R(\mu, v)) \in C(T)$. But $\sigma \cap \tau=\varepsilon_{T}$, so $\tau=\varepsilon_{T}$ or $\tau=\sigma$. The first equality implies that $\mu=\nu$ while the latter is impossible since $R(i, j) \neq \varepsilon$. Thus no two distinct rows of $P$ are identical.

(ii) implies (iii). Suppose that the only distinct identical columns are the $i$ th and $j$ th, and that no two distinct rows are identical. According to Corollary 5, $\sigma=\theta(R(i, j), e, \varepsilon) \in C^{\prime}(T)$. Let $\tau=\theta(r, e, \pi) \in C^{\prime}(T)$. If $\pi \neq \varepsilon$ then it follows easily from Lemma $I$ that $(\varepsilon, e, R(\mu, \nu))$ is an admissible triple for some $\mu \neq \nu$. However this implies that the $\mu$ th and $v$ th rows of $P$ are identical, 
contradicting the hypothesis. Thus $\tau=\theta(r, e, \varepsilon)$, so $r \neq \varepsilon$, which means $k r l$ for some $k \neq l$. But then $(R(k, \tau), e, \varepsilon)$ is an admissible triple, so the $k$ th and $\tau_{\text {th }}$ columns are identical by Corollary 5 . By hypothesis we conclude that $\{k, i\}=\{i, j\}$, so $\tau=\sigma$. Thus $\sigma$ is the only congruence on $T$ different from $\varepsilon$ and $\omega$.

That (iii) implies ( $i$ ) is obvious.

For the remainder of this paper let $S=M^{0}(I, G, M$; P) where $G$ is a nontrivial group and $e$ denotes the identity of $G$. Recall that $\theta_{N}=\theta(\varepsilon, N, \varepsilon)$ for each normal subgroup $N$ of $G$.

PROPOSITION 10. If $S$ is subdirectly irreducible then it is reductive.

Proof. We know from Lemma 2 that $\theta_{G} \in C^{\prime}(S)$. Since $S$ is subdirectly irreducible $\sigma \cap \theta_{G} \neq \varepsilon_{S}$ for all $\sigma \in C^{\prime}(S)$. Thus no triple of the form $(R(i, j), e, \varepsilon)$ or $(\varepsilon, e, R(\mu, v))$ can be admissible since each induced congruence intersects $\theta_{G}$ nontrivially. That $S$ is reductive now follows from Corollary 5 and its dual.

PROPOSITION 11. If $S$ is subdirectly irreducible then $G$ is a subdirectly irreducible group.

Proof. Let $\sigma=\theta(r, N, \pi)$ be the least congruence on $S$. It suffices to show that $\sigma=\theta_{N}$. For in such a case if $K \neq e$ is a normal subgroup of $G$ then $\theta_{K} \in C^{\prime}(S)$ by Lemma 2. But the minimality of $\sigma$ implies that $\theta_{N} \subseteq \theta_{K}$, whence $N \subseteq K$. Thus $N$ is the least normal subgroup of $G$, so $G$ is subdirectly irreducible.

Now we will show that $\sigma=\theta_{N}$. First, suppose that $N=e$; that is, $\sigma=\theta(r, e, \pi)$. If $r \neq \varepsilon$ then $R(i, j) \subseteq r$ for some $i \neq j$, so $(R(i, j), e, \varepsilon)$ is an admissible triple by Lemma 1 . It follows from Corollary 5 that $S$ is not left reductive and from Proposition 10 that $S$ is not subdirectly irreducible, contradicting the hypothesis. The assumption $\pi \neq \varepsilon$ will lead analogously to the same contradiction. Since $\sigma \neq \varepsilon_{S}$ it follows that $N \neq e$, so that $\sigma=\theta_{N}$.

THEOREM 12. A Rees matrix semigroup $S$ over a nontrivial group $G$ 
is subdirectly irreducible if and only if $S$ is reductive and $G$ is subdirectly irreducible.

Proof. In view of Propositions 10 and 11 it suffices to prove the necessity. So let $S$ be reductive and $G$ be subdirectly irreducible with least normal subgroup $K$. We will show that $\theta_{K}$ is the least nonidentical congruence on $S$.

Suppose that $(r, e, \pi)$ is an admissible triple. If $r \neq \varepsilon_{I}$ then $R(i, j) \subseteq r$ for some $i \neq j$, so $\left(R(i, j), e, \varepsilon_{M}\right)$ is admissible by Lemma 1. But Corollary 5 indicates that $S$ is not right reductive, which contradicts the hypothesis. Hence $r=\varepsilon_{I} ;$ similarly $\pi=\varepsilon_{M}$. Therefore no nonidentical congruence on $S$ has the trivial subgroup for its middle entry.

Now let $\sigma \in C^{\prime}(S), \sigma=\theta(r, N, \pi)$. We have seen above that $N \neq e$, so the minimality of $K$ implies that $K \subseteq N$. It is clear that $\theta_{K} \subseteq \sigma$. Finally, Lema 2 insures that $\theta_{K} \neq \varepsilon_{S}$, so $\theta_{K}$ is a nonidentical congruence which is contained in every such congruence.

We might point out that the proofs of the last two results indicate that the least congruence on $S$ is $\theta_{K}$ where $K$ is the least normal subgroup of $G$. Moreover if $\theta(r, N, \pi) \in C^{\prime}(S)$ then $N \neq e$.

\section{References}

[1] Gérard Lallement, "Demi-groupes réguliers", Ann. Mat. Pura Appl. 77 (1967), 47-129.

[2] Gerard Lallement, "A note on congruences on Rees matrix semigroups", Semigroup Forum 8 (1974), 89-92.

[3] Walter Douglas Munn, "Embedding semigroups in congruence-free semigroups", Semigroup Forum 4 (1972), 46-60.

[4] Mario Petrich, Introduction to semigroups (Charles E. Merrill, Columbus, Ohio, 1973).

[5] B.M. Schein [క̌ain], "Homomorphisms and subdirect decompositions of semigroups", Pacific J. Math. 17 (1966), 529-547. 
[9] P.G. Trotter, "Congruence-free inverse semigroups", Semigroup Forum 9 (1974/1975), 109-116.

Department of Mathematics,

Temple University,

Philadelphia,

Pennsylvania,

USA. 